\title{
Comparación de la Calidad de Obturación Radicular, entre el Sistema Termoplastificado Calamus y el Sistema de Compactación Lateral en Frío
}

\author{
Comparison of the Quality of Root Obturation between the \\ Calamus Thermo Plasticized System and the Cold Lateral Compaction
}

Aracena Rojas, D*; Bustos Medina, L. ${ }^{* * * * *}$; Alcántara Dufeu, R. ${ }^{* \star * *}$; Aguilera Pino, O. ${ }^{* * * *}$; Aracena Ghisellini, A. ${ }^{* * * *}$ \& Luengo Pedreros, P. ${ }^{* * * * *}$

ARACENA ROJAS, D.; BUSTOS MEDINA, L.; ALCÁNTARA DUFEU, R.; AGUILERA PINO, O.; ARACENA GHISELLINI, A. \& LUENGO PEDROSO, P. Comparación de la calidad de obturación radicular, entre el sistema termoplastificado Calamus y el sistema de compactación lateral en frío. Int. J. Odontostomat., 6(2):115-121, 2012.

RESUMEN: El objetivo de este trabajo fue establecer las diferencias en la calidad de la obturación radicular entre la técnica termoplastificada Calamus y la técnica de compactación lateral en frío, cuando se utilizan conos de gutapercha protaper universal. Se realizó una investigación cuasi-experimental in vitro, con una muestra no probabilística de 80 piezas dentarias unirradiculares, cuyos canales fueron instrumentados con el sistema Protaper Universal Manual. Posteriormente se dividieron aleatoriamente en dos grupos de 40 raíces cada uno. El grupo uno fue obturado con el sistema Calamus, y el grupo dos con el sistema de compactación lateral. Las muestras fueron sometidas a la prueba de filtración apical por difusión de tintes, diafanizadas y cortadas transversalmente para su evaluación radiográfica y microscópica. Los datos fueron analizados con la prueba exacta de Fisher. El sistema de compactación lateral obtuvo un sellado más eficiente de los canales radiculares que el sistema Calamus $(p=0,229)$. El sistema termoplastificado presentó una mejor adaptación de la masa de gutapercha sólo a nivel del tercio medio $(p=0,037)$ y una menor presencia de vacuolas en todo el canal $(p=0,020)$, diferencias estadísticamente significativas. Además, el sistema Calamus presentó una mayor cantidad de canales accesorios obturados y de raíces filtradas, pero estas diferencias no fueron significativas. El sistema de compactación lateral demostró ser más eficiente que la técnica termoplastificada Calamus, pero estas diferencias no son significativas.

PALABRAS CLAVE: endodoncia, obturación canal radicular, terapia canal radicular.

\section{INTRODUCCIÓN}

El tratamiento del canal radicular consiste en la eliminación completa de la pulpa, la limpieza y correcta conformación del canal, para finalizar con la obturación, con el fin de que se pueda conservar el diente como una unidad funcional del sistema estomatognático.

La filtración entre el material de obturación y las paredes del canal afectan de forma negativa el éxito del tratamiento endodóntico, por esta razón el éxito radica en que el material de obturación logre un buen sellado apical y llene en forma tridimensional todo el lumen del canal (Echeverría \& Pumarola, 1995).

La causa principal de inflamación periapical persistente y de los fracasos endodónticos, corresponderían a la filtración del suero desde los tejidos apicales, proporcionando nutrientes a los microorganismos remanentes que se encuentran en los túbulos del canal radicular. En las últimas décadas los métodos de filtración han empleado colorantes, radioisótopos, bacterias y sus metabolitos. Pero el método más utilizado por su

\footnotetext{
Facultad de Odontología, Departamento Integral del Adulto, Universidad de La Frontera, Temuco, Chile.

** Centro de Excelencia CIGES (Capacitación, Investigación y Gestión para la Salud Basada en la Evidencia), Facultad de Medicina, U. de La Frontera, Temuco.

*** Dpto. Salud Pública, Facultad de Medicina, U. de La Frontera, Temuco, Chile.

${ }^{* * * *}$ Facultad de Odontología, Universidad de Concepción, Concepción, Chile.

${ }^{* * * * *}$ Cirujanos Dentistas, Universidad Mayor, Temuco, Chile.
} 
facilidad, sensibilidad y conveniencia es la penetración de tintes, ya que nos indicaría el espacio que queda entre la pared del canal y el material obturador (Wimonchit et al., 2002).

La técnica de compactación lateral en frío es la técnica más utilizada por su eficacia comprobada, sencillez, control del límite apical de la obturación y el uso de instrumental simple. Pérez et al. (2007), señalan que, para mejorar el relleno del canal se han desarrollado conos maestros que se adaptan a canales preparados con instrumentos rotatorios o manuales de niquel-titanio de conicidad aumentada $0,04 \%$ y $0,06 \%$, a diferencia de la compactación lateral que presenta conicidades de $0,02 \%$. Combinar ambas técnicas permite obtener una mejor calidad de obturación con un relleno de gutapercha más denso mediante una técnica sencilla.

La obturación de los canales radiculares ha mejorado indudablemente en el tiempo, gracias a la introducción de nuevas tecnologías en la especialidad, las cuales han contribuido a lograr una adaptación y sellado más eficiente del sistema de canales radiculares.

La técnica de termoplastificado de onda continua desarrollada por Calamus, es una innovación que ofrece a los profesionales de la odontología una herramienta con la que podrían obtener, una mejor calidad de obturación que los métodos convencionales. Gilhooly et al. (2000), muestra en sus estudios que la filtración de los canales obturados con técnicastermoplastificadas fue significativamente menor que con la técnica de compactación lateral en frío $(p<0,05)$. Los estudios realizados por De-Deus et al. (2006), respecto de la adaptación de gutapercha en el tercio apical muestran una mejor adaptación con el sistema termoplastificado $(85,7 \%)$ que con la técnica de compactación lateral $(82,6 \%)$

El objetivo de este trabajo fue establecer las diferencias en la calidad de la obturación radicular, entre la técnica termoplastificada de onda continua Calamus y la técnica de compactación lateral en frío, cuando se utilizan conos de gutapercha protaper universal.

\section{MATERIAL Y MÉTODO}

Estudio cuasi-experimental en 112 piezas dentarias humanas extraídas por razones periodontales y por caries, sin posibilidad de restauración, las que fueron almacenas y sumergidas en una solución de clorhexidina al $0,12 \%$ hasta el comienzo del estudio.
Las piezas seleccionadas cumplieron los siguientes criterios de inclusión: unirradiculares, formación apical completa, canales sin calcificaciones, sin rizálisis, sin fractura radicular y que presentaran curvatura radicular inferior a $30^{\circ}$. Del total, fueron seleccionadas 80 piezas dentarias, las que fueron sometidas a una limpieza por medio de puntas de ultrasonido Endo Proultra de zirconio en un equipo marca Satelec y de una cureta $7 / 8$, Gracey- Hu-Friedy, para eliminar restos de ligamento periodontal de la superficie radicular. Luego fueron conservadas durante 48 horas en una solución de hipoclorito de sodio al $5,25 \%$ a efecto de eliminar restos de tejidos orgánicos remanentes.

A cada pieza dentaria se les amputaron las coronas a nivel de la unión amelocementaria y se permeabilizaron sus canales con una lima $\mathrm{K} \mathrm{N}^{\circ} 10$ (Dentsply-Maillefer $囚)$. Posteriormente, todas las raíces dentarias fueron almacenadas y numeradas en tubos de ensayo individuales, inmersos en una solución de clorhexidina al $0,12 \%$.

Las muestras fueron manipuladas por dos operadores distintos previamente entrenados, con la finalidad de sesgar con respecto a los grupos de obturación y a quien recopile los resultados. El operador 1 realizó la determinación de la longitud de trabajo, la instrumentación biomecánica, interpretó resultados radiográficos, los resultados de filtración en piezas diafanizadas y resultados de adaptación de la masa de gutapercha en cortes transversales de dientes diafanizados. El operador 2 realizó la obturación aleatoria de cuarenta piezas por cada sistema de obturación, la técnica de filtración apical, la diafanización y la toma radiográfica mediante la técnica del paralelismo con una película ultraspeed DF 57 Kodac, utilizando una mesa de trabajo que permitió estandarizar la técnica. Todas las piezas se ubicaron en forma paralela al eje longitudinal de las raíces y a una distancia estandarizada de $47,2 \mathrm{~cm}$. La potencia correspondió a $70 \mathrm{Kv}$ en modo adulto y las placas radiográficas se revelaron en un revelador automático Perio Mat Plus $₫$.

La longitud de trabajo se obtuvo con una lima K10 (Dentsply-Maillefer®), la cual fue introducida en cada canal hasta que la punta de la lima emergiera por el borde del foramen apical, restando $0,5 \mathrm{~mm}$ para corresponderse con la ubicación aproximada de la constricción apical.

El operador 1 instrumentó las ochenta muestras con limas del sistema ProTaper Universal Manual (Dentsply-Maillefer®). Previa permeabilización del ca- 
nal con una lima K10 (Dentsply-Maillefer®), se trabajó el tercio cervical con la lima SX, continuando con la lima S1-S2-F1-F2-F3 hasta la longitud de trabajo, instrumentando previamente con Limas $\mathrm{K} \mathrm{N}^{\circ} 20-25$ o 30 , según el calibre apical de la lima protaper a utilizar. Además entre cada lima se realizó una irrigación profusa con $15 \mathrm{cc}$ de hipoclorito de sodio al 5,25\%, mediante una jeringa Plastipack con aguja BD Precision/Glide (0,60x1") y un acondicionador de las paredes dentinarias con EDTA gel al 17\% Glyde (Dentsply-Maillefer®).

Las raíces dentarias fueron asignadas a dos grupos de estudio, elegidos al azar. El grupo 1 compuesto por cuarenta muestras, fueron obturadas con conos protaper universal, mediante el sistema Calamus (Dentsply-Maillefer ${ }^{\circledR}$ ) y el grupo 2 compuesto por cuarenta muestras obturadas con conos protaper universal, mediante el sistema de compactación lateral. Finalmente se obtuvieron las radiografías de control respectivas, mediante una mesa de trabajo especialmente diseñada para estandarizar la técnica.

Protocolo de Obturación Sistema Calamus. Irrigación con hipoclorito de sodio al $5 \%$ y secado con conos de papel (Dentsply-Maillefer ${ }^{\circledR}$ ), del sistema de canales previamente instrumentado.

Prueba de ajuste del cono maestro Protaper a LT. Se consideró óptimo, cuando presentó cierta resistencia para su retiro del canal. Se impregna el cono maestro de gutapercha, con una mínima cantidad de cemento sellador Top Seal (Dentsply-Maillefer () ), para luego ubicarlo a LT en el canal.

Cortamos el cono a nivel de la entrada del canal con el sistema Pack del Calamus a $200^{\circ} \mathrm{C}$ y condensamos manualmente. Posteriormente, llevamos la gutapercha con el Pack a - $4 \mathrm{~mm}$ de la LT. y compactamos con un condensador manual marca Sybron Endo Buchanam $N^{\circ} 1$.

Con el sistema Flow de Calamus a $200^{\circ} \mathrm{C}$, se depositan aproximadamente $3 \mathrm{~mm}$ de gutapercha termoplástica compactándola verticalmente con un condensador marca Sybron Endo Buchanam №2 ó 3, de acuerdo con el calibre del canal. Este procedimiento se repite hasta lograr el relleno total del sistema de canales.

Protocolo de Obturación de Compactación Lateral en Frío. Irrigación con hipoclorito de sodio al 5\% y secado con conos de papel (Dentsply-Maillefer $\left.{ }^{\circledR}\right)$, del sis-

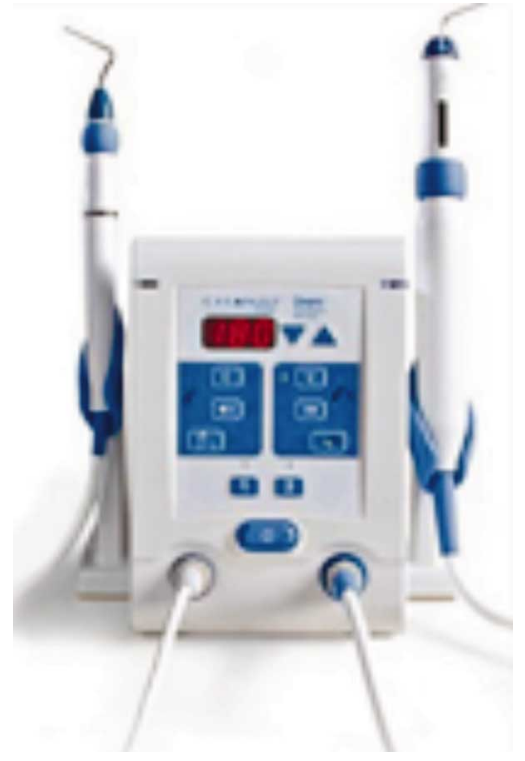

Fig.1 Unidad Calamus.

tema de canales previamente instrumentado. Prueba de ajuste del cono maestro Protaper a LT. Se consideró óptimo, cuando presentó cierta resistencia para su retiro del canal.

Mediante el cono maestro, se impregnan las paredes del canal, con una moderada cantidad de cemento sellador Top Seal (Dentsply-Maillefer®), rotándolo en sentido antihorario hasta ubicarlo a LT.

Mediante un condensador digital $n^{\circ} 30$ (DentsplyMaillefer $($ ) se procede a compactar en sentido vestíbulo lingual o palatino el cono maestro con un espaciador $\mathrm{n}^{\circ} 30$, colocando conos accesorios $\mathrm{N}^{\circ} 25$ (DentsplyMaillefer®), en aquellos canales que por su anatomía, no fue posible obtener un relleno completo al utilizar la técnica del cono único que recomienda el fabricante.

Una vez insertado el último cono accesorio, se procedió a cortarlos con un condensador calentado al rojo cereza y se condensó en forma vertical.

Preparación de las Raíces. Las raíces dentales de los dos grupos fueron cubiertas con barniz de uña hasta 5 $\mathrm{mm}$ del ápice, sumergidas en una solución de tinta china y calentadas en baño termorregulador con una humedad del $100 \%$ a una temperatura de $37^{\circ} \mathrm{C}$, simulando la temperatura bucal normal por 48 horas. Luego fueron retiradas del baño para ser sometidas al proceso de diafanización dentaria la que permite transformar un diente natural en uno transparente total para su análisis (Mullaney et al., 1979). 
Posteriormente fueron sumergidas en ácido clorhídrico al $7 \%$ durante 48 horas para su descalcificación. Transcurrido este tiempo, se sumergieron en alcohol al $70 \%$ durante 5 horas, en las siguientes 5 horas en alcohol al $80 \%$, luego 5 horas en alcohol al $96 \%$ y por último en alcohol al $100 \%$ otras 5 horas. Este procedimiento deshidrató los dientes, para luego colocarlos en salicilato de metilo completándose la transparentación en 24 a 48 horas (Paredes et al., 1993; Lozano, 2004).

Luego las muestras de los dos grupos de estudio fueron observadas y medidas con una lupa binocular microscópica marca Arquimed, a 20x y 40x. Estas imágenes fueron directamente capturadas a través de los oculares de la Lupa por medio de una cámara fotográfica digital (Cyber-shot - Sony $®)$, las que se transfirieron a un computador por medio de un puerto USB. Se procedió a observar la presencia de canales accesorios obturados y la existencia de sobre o subobturaciones (Fig. 2 ), y el grado de filtración de la gutapercha (Fig. 3) a partir del extremo más apical del cono , hasta la zona de mayor tinción hacia coronal.

La medición de la subobturación y de la extensión de la filtración se realizó por medio de las imágenes fotográficas de cada pieza dentaria, siendo analizadas con el software Motic Images Plus $2.0{ }^{\circledR}$.

Finalmente a cada raíz se le efectuó un corte transversal por medio de un bisturí, a los $3 \mathrm{~mm}$ y 7 $\mathrm{mm}$ del ápice. Cada porción de la raíz fue observada por medio de una lupa binocular, para analizar la adaptación de la masa de gutapercha y la presencia de vacuolas al interior de la masa obturadora. Independientemente se evaluó la presencia de oquedades en las radiografías de control obtenidas luego de que las raíces fueron obturadas.
Se interpretó como éxito de la obturación radicular, cuando no se hicieron presentes en el sellado de las muestras variables como, filtración apical, vacuolas y desadaptación de la masa de gutapercha.

Análisis estadístico. Todos los datos registrados fueron ingresados en una ficha Excel 2007. Luego, fueron procesados estadísticamente con el programa Stata v11.0, realizándose un análisis descriptivo a través de frecuencias y porcentajes. Para la comparación de los porcentajes se utilizó la prueba exacta de Fisher, el nivel de significación fue de $5 \%$.

\section{RESULTADOS}

Al comparar la calidad del sellado apical entre las dos técnicas, se encontró que el sistema Calamus presentó una menor presencia de vacuolas en la masa obturadora $(p=0,020)$ y una mayor adaptación de la masa de gutapercha a nivel del tercio medio $(p=0,037)$ que la técnica de compactación lateral, diferencias estadísticamente significativas. Por otra parte, el sistema termoplastificado muestra una mayor cantidad de canales accesorios obturados y de raíces filtradas, pero estas diferencias no fueron significativas.

Al considerar el número de fallas, se encontró un mayor porcentaje de canales sellados herméticamente con la técnica de compactación lateral $77,5 \%$, respecto de Calamus $60 \%$, pero estas diferencias no fueron estadísticamente significativas $p=0,229$ (ver Tabla I).

En lo que respecta a la presencia de oquedades radiográficas, el mayor porcentaje se encuentra en el grupo Calamus, por sobre el grupo de

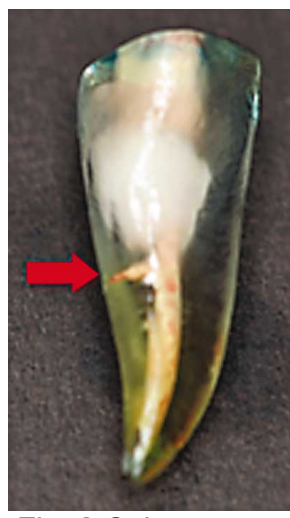

Fig. 2 Calamus, canal accesorio.

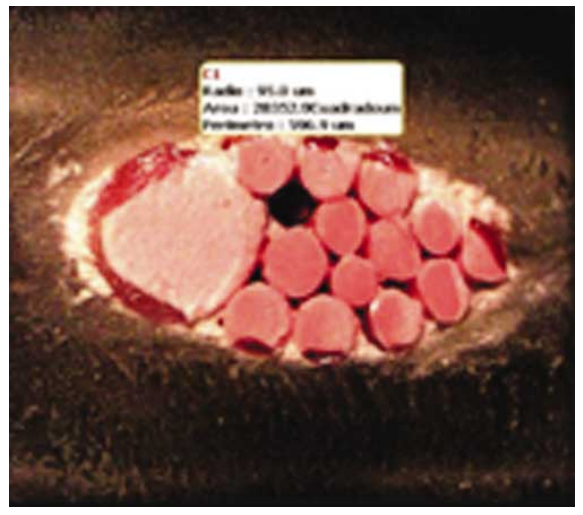

Fig. 3 Compactación lateral.

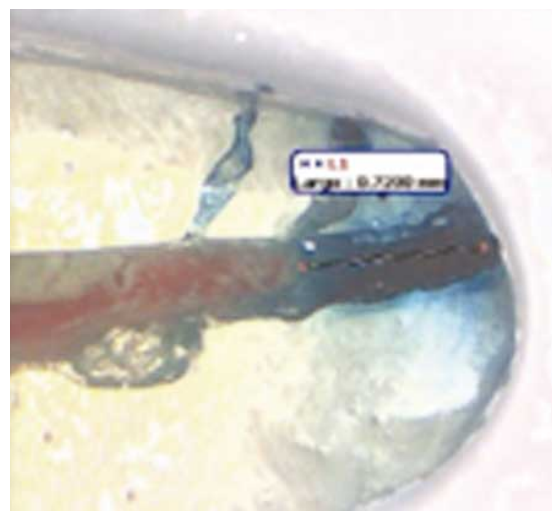

Fig. 4 Filtración apical. 
compactación lateral, diferencias que no fueron significativas.

Al examinar la extensión de la obturación radicular, a través de una lupa y mediante radiografías post diafanización de las raíces, no se encontraron diferencias estadísticamente significativas.

\section{DISCUSIÓN}

La obturación de los canales radiculares ha mejorado en el tiempo, gracias a la introducción de nuevas tecnologías, que han contribuido a lograr un sellado más eficiente del sistema de canales radiculares, pero el sistema de compactación lateral sigue presentándose como una alternativa efectiva.
Valli et al. (1998) \& Cobankara et al. (2000) señalaron que el sellado apical se mide y demuestra "in vitro" mediante técnicas de microfiltración apical, siendo el método más común el de difusión de tintes a lo largo de la raíz, el cual se puede observar mediante cortes o diafanización, lo que permite la observación del colorante en tres dimensiones. El tinte utilizado en este estudio fue tinta china, porque de acuerdo con Ponce et al. (2005) no es afectado por el proceso de diafanización.

Las bacterias residuales que sobreviven en el canal luego de su limpieza y conformación, pueden llevar al fracaso del tratamiento endodóntico, de ahí la importancia de un óptimo sellado, el cual actuará impidiendo la interacción de las bacterias residuales con los tejidos perirradiculares (Ponce et al.).

Tabla I. Variables de calidad de obturación radicular para las técnicas Termoplastificado Calamus y Condensación Lateral en Frío.

\begin{tabular}{|c|c|c|c|c|c|c|c|}
\hline & & \multicolumn{2}{|c|}{ Calamus } & \multicolumn{2}{|c|}{ C. Lateral } & \multicolumn{2}{|l|}{ Total } \\
\hline & & Frec. & $\%$ & Frec. & $\%$ & $\%$ & $\mathbf{p}$ \\
\hline \multirow{2}{*}{ Filtración Apical } & Ausencia & 36 & 90,0 & 37 & 9,5 & 91,3 & \multirow{2}{*}{1,000} \\
\hline & Presencia & 4 & 10,0 & 3 & 7,5 & 8,8 & \\
\hline \multirow{2}{*}{ Vacuolas } & Ausencia & 37 & 92,5 & 28 & 70,0 & 81,3 & \multirow{2}{*}{0,020} \\
\hline & Presencia & 3 & 7,5 & 12 & 30,0 & 18,8 & \\
\hline \multirow{3}{*}{ Desad. Apical } & Ausencia & 35 & 87,5 & 35 & 87,5 & 87,5 & \multirow{2}{*}{1,000} \\
\hline & Presencia & 5 & 12,5 & 5 & 12,5 & 12,5 & \\
\hline & 0 & 24 & 60,0 & 31 & 77,5 & 68,8 & \multirow{3}{*}{0,229} \\
\hline \multirow[t]{2}{*}{$N^{\circ}$ de fallas } & 1 de tres & 12 & 30,0 & 6 & 15,0 & 22,5 & \\
\hline & 2 de tres & 4 & 10,0 & 3 & 7,5 & 8,8 & \\
\hline \multirow{2}{*}{ Desad. Tercio Medio } & Ausencia & 37 & 92,5 & 29 & 72,5 & 82,5 & \multirow{2}{*}{0,037} \\
\hline & Presencia & 3 & 7,5 & 11 & 27,5 & 17,5 & \\
\hline \multirow{3}{*}{$\begin{array}{l}\text { Ext. Obt. Radicular } \\
\text { Visión directa }\end{array}$} & Correcta & 33 & 82,5 & 32 & 80,0 & 81,3 & \multirow{3}{*}{0,835} \\
\hline & Subobt & 3 & 7,5 & 5 & 12,5 & 10,0 & \\
\hline & Sobreobt & 4 & 10 & 3 & 7,5 & 8,8 & \\
\hline \multirow{3}{*}{$\begin{array}{l}\text { Ext. Obt. Radicular } \\
\text { Radiográfica }\end{array}$} & Correcta & 34 & 85,0 & 27 & 67,5 & 76,3 & \multirow{3}{*}{0,136} \\
\hline & Subobt & 6 & 15 & 11 & 27,5 & 21,3 & \\
\hline & Sobreobt & 0 & 0 & 2 & 5,0 & 5,0 & \\
\hline \multirow{2}{*}{ Cal. Ob. Visión Lupa } & Ausencia & 34 & 85,0 & 39 & 97,5 & 91,3 & \multirow{2}{*}{0,108} \\
\hline & Presencia & 6 & 15,0 & 1 & 2,5 & 8,8 & \\
\hline \multirow{2}{*}{ Cal. Ob. Visión Rx } & Ausencia & 30 & 75,0 & 37 & 92,5 & 83,8 & \multirow{2}{*}{0,660} \\
\hline & Presencia & 10 & 25,0 & 3 & 7,5 & 16,3 & \\
\hline \multirow{2}{*}{ Oquedades } & Ausencia & 34 & 85,0 & 38 & 95,0 & 90,0 & \multirow{2}{*}{0,263} \\
\hline & Presencia & 6 & 15,0 & 2 & 5,0 & 10,0 & \\
\hline
\end{tabular}

Prueba Exacta de Fisher. Ca. Ob. = Canales Accesorios Obturados; Cal. Ob. = Calidad de la Obturación, Ext. Ob. = Extensión Obturación; Des. = Desadaptación; № Fallas= Desadaptación, oquedad, filtración. 
En el presente trabajo, al comparar la filtración apical entre el sistema Calamus respecto de la compactación lateral en frío, no se encontraron diferencias significativas entre ambos métodos $(p=1,000)$, a diferencia de lo que encontró Gilhooly et al., donde el grado de filtración de los canales obturados con técnicas termoplastificadas fue significativamente menor que la técnica de condensación lateral $(p<0,050)$.

Otra variable analizada correspondió a la adaptación de la masa de gutapercha a nivel del tercio apical y medio del canal radicular. Los resultados obtenidos en este estudio mostraron valores similares para la adaptación en el tercio apical con un $87,5 \%$ en cada una de las técnicas. De igual forma, los estudios de De-Deus et al. muestran un $82,6 \%$ de adaptación en el tercio apical para la técnica de compactación lateral y un $85,7 \%$ para el sistema termoplastificado. A nivel del tercio medio, nuestro estudio muestra una mayor adaptación con la técnica Calamus $(92,5 \%)$ que la técnica de compactación lateral $(72,5 \%)$, siendo esta diferencia estadísticamente significativa $p=0,037$.

Respecto a la presencia de vacuolas en la masa obturadora, en este estudio se encontraron más vacuolas en la técnica de compactación lateral que la técnica Calamus, diferencias que fueron significativas $p=0,020$. Por el contrario, De-Deus et al., afirman que no hay diferencias estadísticamente significativa en la adaptación a nivel del tercio medio, confirmando una adaptación de $85,6 \%$ para la compactación lateral y del $88,2 \%$ para la técnica termoplastificada.

\section{En contraposición a estudios in vitro de}

Gulabivala et al. (1998), donde compararon la técnica de compactación lateral y otras técnicas termoplastificadas, como Thermafil $₫$ y JS Quick Fill $囚$ muestran que la calidad de la obturación radicular se vio afectada por la extrusión de cemento sellador o gutapercha, en forma significativa $(p=0,020)$, en este estudio no se encontró mayor cantidad de casos con sobreobturación radicular con la técnica termoplastificada Calamus, respecto de la técnica de compactación lateral en frío $(p=0,835)$.

Al igual que los trabajos de Zielinski et al. (2008) y Karabucak et al. (2008), que muestran la efectividad de las técnicas termoplastificadas para obturar canales accesorios, en este estudio se encontró un elevado porcentaje de canales accesorios obturados, tanto a visión microscópica como radiográfica; $15 \%$ y $25 \%$ respectivamente, sin embargo, no constituyen resultados estadísticamente significativos, debido tal vez al tamaño de la muestra, a la anatomía de las pieza dentaria o las propiedades viscoelásticas de la gutapercha.

Los sistemas termoplastificados de obturación radicular, no han logrado demostrar mayor eficacia clínica que los sistemas de obturación en frío, de ahí que un porcentaje importante de Odontólogos sigue utilizando la técnica de compactación lateral.

En conclusión, al considerar el número de fallas, el sistema de compactación lateral demostró ser más eficiente que la técnica Calamus al obtener un mayor porcentaje de sellado hermético de los canales radiculares, pero estas diferencias no son estadísticamente significativas.

ARACENA ROJAS, D.; BUSTOS MEDINA, L.; ALCÁNTARA DUFEU, R.; AGUILERA PINO, O.; ARACENA GHISELLINI, A. \& LUENGO PEDROSO, P. Comparison of the quality of root obturation between the Calamus thermo plasticized system and the cold lateral compaction. Int. J. Odontostomat., 6(2):115-121, 2012.

ABSTRACT: The aim of this work was to establish the differences in the quality of the root obturation between the Calamus thermo plasticized gutta-percha technique, and the cold lateral compaction technique, when universal protaper gutta percha cones are used. A quasi-experimental "in vitro" investigation was developed, using a not probabilistic sample of 80 single rooted teeth whose conducts were instrumented by the Manual Protaper Universal system. They where then randomly divided in two groups of 40 each. The first group was filled with Calamus system and the second group with the lateral compaction system. The samples were submitted to the test of apical filtration for diffusion of dyes, diafanized and cut transversely for radiographic and microscopic evaluation. The information was analyzed by Fisher's exact test. The lateral compaction technique obtained a more efficient seal of the root canals than the Calamus system $(p=0.229)$. On the other hand the Calamus thermo platicized system presented a better adjustment of the gutta-percha mass, only at the middle third $(p=0.037)$ and a minor presence of vacuoles in the whole conduct $(p=0.020)$, being both statistically significant differences. In addition, the Calamus system, presented both, a major quantity of accessory canals filled and a mayor number of leaked roots, but these differences were not significant. The system of lateral compaction demonstrated to be more efficient than the Calamus thermo plasticized technique, but these differences were not significant.

KEY WORDS: endodontic, root canal obturation, root canal therapy. 
ARACENA ROJAS, D.; BUSTOS MEDINA, L.; ALCÁNTARA DUFEU, R.; AGUILERA PINO, O.; ARACENA GHISELLINI, A. \& LUENGO PEDROSO, P. Comparación de la calidad de obturación radicular, entre el sistema termoplastificado Calamus y el sistema de compactación lateral en frío. Int. J. Odontostomat., 6(2):115-121, 2012.

\section{REFERENCIAS BIBLIOGRÁFICAS}

Cobankara, F. K.; Adanir, N.; Belli, S. \& Pashley, D. H. A quantitative evaluation of apical leakage of four root-canal sealers. Int. Endod. J., 35(12):979-84, 2002.

De-Deus, G.; Gurgel-Filho, E.; Magalhaes, K. \& Coutinho, T. A laboratory analysis of gutta-perchafilled area obtained using Thermafil, System B and lateral condensation. Int. Endod. J., 39(5):37883,2006 .

Echeverría, J. \& Pumarola, J. EL Manual de Odontología. $2^{\mathrm{a}}$ Ed. Barcelona, Masson S.A., 1995.

Gilhooly, R. M.; Hayes, S. J.; Bryant, S. T. \& Dummer, P. M. Comparison of cold lateral condensation and a warm multiphase gutta-percha technique for obturating curved root canals. Int. Endod. J., 33(5):415-20, 2000.

Gulabivala, K.; Holt, R. \& Long, B. An in vitro comparison of thermoplasticised gutta-percha obturation techniques with cold lateral condensation. Endod. Dent. Traumatol., 14(6):2629, 1998.

Karabucak, B.; Kim, A.; Chen, V. \& Iqbal, M. K. The comparison of gutta-percha and Resilon penetration into lateral canals with different thermoplastic delivery systems. J. Endod., 34(7):847-9, 2008.

Lozano, A. A.; Forner Navarro, L. \& Llena Puy, M. C. Estudio in vitro de la anatomía del sistema de canales radiculares. Revista de Endodoncia, 22(4): 236-43,2004

Mullaney, T. P. Instrumentation of finely curved canals. Dent. Clin. North Am., 23(4):575-92, 1979.

Paredes, J.; Howard, A. \& Guizar, C. A. Diafanización dental, una alternativa por la enseñanza preclínica en endodoncia. Práctica Odontológica, 14(9):9-10, 1993.

Pérez Heredia, M.; Clavero González, J.; Ferrer Luque, C. M. \& González Rodríguez, M. P. Apical seal comparison of low-temperature thermoplasticized gutta-percha technique and lateral condensation with two different master cones. Med. Oral Patol. Oral Cir. Bucal, 12(2):E175-9, 2007.
Ponce, A.; Izquierdo, J. C.; Sandoval, F. \& De los Reyes, J. Estudio comparativo de la filtración apical entre la técnica de compactación lateral en frío y técnica de obturación con System B. Rev. Odont. Mex., 9(2):65-72, 2005.

Valli, K. S.; Rafeek, R. N. \& Walker, R. T. Sealing capacity in vitro of thermoplasticized gutta-percha with a solid core endodontic filling technique. Endod. Dent. Traumatol., 14(2):68-71, 1998.

Wimonchit, S.; Timpawat, S. \& Vongsavan, N. A comparison of techniques for assessment of coronal dye leakage. J. Endod., 28(1):1-4, 2002.

Zielinski, T. M.; Baumgartner, J. C. \& Marshall, J. G. An evaluation of Guttaflow and gutta-percha in the filling of lateral grooves and depressions. J. Endod., 34(3):295-8, 2008.

\author{
Dirección para Correspondencia: \\ Sergio Daniel Aracena Rojas \\ Depto. Odontología Integral \\ Manuel Montt №112 Of. 420 \\ Temuco \\ CHILE \\ Fono-fax: (45) 325775 \\ Email: saracena@ufro.cl
}

Recibido : 21-01-2012

Aceptado: 26-05-2012 